\title{
Russie : les paradoxes de la violence (Partie 2)
}

\section{Anne Le Huerou}

\section{(2) OpenEdition}

Journals

\section{Édition électronique}

URL : http://journals.openedition.org/conflits/694

DOI : $10.4000 /$ conflits.694

ISSN : $1777-5345$

Éditeur :

CCLS - Centre d'études sur les conflits lilberté et sécurité, L'Harmattan

\section{Édition imprimée}

Date de publication : 15 octobre 1998

ISSN : 1157-996X

\section{Référence électronique}

Anne Le Huerou, «Russie : les paradoxes de la violence (Partie 2) », Cultures \& Conflits [En ligne], 29-30 | automne-hiver 1998, mis en ligne le 16 mars 2006, consulté le 30 mars 2021. URL : http:// journals.openedition.org/conflits/694; DOI : https://doi.org/10.4000/conflits.694

Ce document a été généré automatiquement le 30 mars 2021.

Creative Commons License 


\title{
Russie : les paradoxes de la violence (Partie 2)
}

\author{
Anne Le Huerou
}

La violence de l'Etat peut aussi venir de l'action arbitraire et incontrôlée d'institutions héritées d'un système non démocratique, où les vraies instances de décision ne sont pas élues, et où l'absence de contrôle mène facilement à l'arbitraire et à la violence. Ainsi en est-il du Conseil de Sécurité, organisme censé veiller sur les intérêts supérieurs de l'Etat, mission peu compatible avec la transparence et le contrôle démocratique, et dont le rôle n'a cessé de croître depuis son institution en mars 1992. L'acception du terme sécurité y est très étendue, ce qui peut autoriser le Conseil à former une sorte de directoire des affaires du pays. L'enjeu qu'a représenté en juin 1996 la nomination d'Alexandre Lebed au poste de Secrétaire général de ce conseil indique bien l'étendue des pouvoirs accordée à cette fonction. Quant aux services de sécurité, ex-KGB devenu FSB, ils cumulent les expressions de violence politique et économique, et sont accusés de connexions avec le crime organisé ${ }^{1}$ et de nombreuses violations des droits de l'homme $^{2}$.

2 Conséquence directe de la crise financière et de la crise de commandement de l'armée, la " criminalité en galons " a augmenté de $25 \%$ en 1995, avec une augmentation de $76 \%$ pour les crimes graves et un doublement des meurtres. Si la majorité des crimes sont commis par les appelés, de plus en plus sont le fait d'officiers. Les crimes et délits les plus courants sont l'abus d'autorité, la corruption, le trafic et la revente d'armes et de munitions. La réforme de l'armée est constamment freinée et le maintien de structures doubles voire multiples est souvent considérée comme une menace pour la démocratie ${ }^{3}$. Les liens entre forces armées et crime organisé sont de plus en plus manifestes ${ }^{4}$.

3 La criminalité à l'intérieur de l'armée correspond-elle à des comportements susceptibles de basculer dans la violence politique, par exemple dans un coup d'Etat des officiers non soldés depuis des mois, ainsi que le prédisent régulièrement des hauts responsables militaires, exhortant l'Etat à faire face à ses obligations ? Ou bien relève-telle de significations classiques, que la position et les ressources de ses auteurs rendent 
plus spectaculaires et objectivement plus déstabilisatrices pour l'ensemble de la société?

4 L'héritage soviétique en ce domaine favorise les tendances centrifuges, avec la dispersion des appartenances et des commandements des forces de l'ordre comme des forces armées. Les logiques historiques, stratégiques ou politiques qui ont présidé à cette disposition ne sont plus pertinentes, ni opérationnelles aujourd'hui. Il n'en subsiste que dispersion et confusion. Le ministère de l'Intérieur (MVD) a la particularité d'avoir sous ses ordres, outre les forces de police " classiques " (Militsia) et des unités spéciales d'intervention (les OMON, créées dans les tout derniers moments de l'Union soviétique et utilisées pour des interventions dans les conflits liés à l'éclatement du pays), des unités militaires en nombre non négligeable. Celles-ci ont été particulièrement impliquées dans la guerre en Tchétchénie, ce qui permettait d'autant plus facilement aux autorités russes de tenir le discours d'une " affaire intérieure " réglée par des forces relevant du maintien de l'ordre, surtout après la nomination du Général Andreï Koulikov, un " transfuge " de l'armée, à la tête du MVD. Ceci complique l'analyse des acteurs impliqués dans le conflit tchétchène, comme d'ailleurs d'autres conflits armés de la région. La Russie y est impliquée de manière ambiguë, officiellement comme force de maintien de la paix entre deux parties en conflit, mais apparaît le plus souvent comme soutenant l'une des parties. Le conflit abkhaze est un bon exemple de cette ambiguïté, mais l'on pourrait aussi citer le cas du Tadjikistan. A la multiplicité des acteurs partie prenante (corps de gardes-frontières dépendant du ministère de la Défense avec un commandement distinct, mais aussi OMON relevant du MVD), s'ajoutent les incohérences, voire les divergences ou les rivalités qui opposent leurs commandements respectifs. Cette situation rend particulièrement peu lisible le rôle de la Russie dans les conflits qui affectent le " proche étranger "5, et elle donne le sentiment général que l'armée en tant que telle est marginalisée.

5 Il faut ajouter à ce tableau les forces spéciales relevant du FSB (ex-KGB pour ce qui relève du territoire de la Russie), et notamment les commandos Alpha qui s'étaient illustrés en août 1991 en refusant de donner l'assaut à la Maison Blanche, et les différentes " gardes " dont la plus célèbre est la garde présidentielle, que le Général Korjakov, un ancien proche de Boris Eltsine aujourd'hui en disgrâce, a amené à un effectif de cinquante mille hommes. On peut aussi mentionner les milices bancaires ${ }^{6}$, dont les liens sont directs et permanents avec les forces précédemment citées, et qui marquent un pas de plus dans la privatisation.

Cette situation est lourde de conflits et de rivalités qui basculent régulièrement dans la violence. On peut citer, par exemple, deux épisodes de la guerre des polices ou des services. En décembre 1994, des membres de la Garde présidentielle, cagoulés et méconnaissables, prennent d'assaut l'une des banques privées les plus importantes de la capitale, très liée au maire, la Most Bank, et tirent ensuite sur des forces de police ainsi que sur des agents du FSB venus sur les lieux et croyant à une opération criminelle. Quelques mois plus tard, un échange de coups de feu entre policiers et agents du FSB faisait un mort et deux blessés graves dans la rue, en plein jour, à Moscou. Les deux incidents ont des origines différentes : dans le premier cas, le Général Korjakov a utilisé dans une stratégie à double détente des forces publiques pour régler avec une institution privée des comptes qui n'avaient sans doute directement à voir ni avec l'une ni avec l'autre. L'incident traduisait plutôt une lutte pour le contrôle des médias dont l'enjeu principal était, à ce moment-là, une rivalité sans merci entre le 
Général Korjakov et Vladimir Gussinski, patron de la chaîne indépendante NTV, en partie financée par la Most Bank. Dans le second cas, l'affaire relève peut-être plus de la bavure, mais n'en est pas moins révélatrice des rivalités et de la désorganisation qui règnent.

7 Cette tendance à la privatisation, à l'apparition d'une multitude de forces paramilitaires, de mercenaires, de " miliciens, condottieri et brigands "7 est beaucoup plus importante dès que l'on dépasse les frontières de la Russie vers le Caucase ou de l'Asie Centrale. Néanmoins, le rôle que continue d'y jouer la Russie, y compris et surtout sur le plan militaire, impliquait au moins de le mentionner. Dans de nombreux conflits de ces dernières années, les forces armées russes, censées maintenir la paix, ont souvent contribué à la guerre, que ce soit par des ventes d'armes et de munitions par les unités de l'ex-armée soviétique auparavant stationnées dans ces Républiques, ou par le soutien d'unités militaires à l'une au l'autre des parties, parfois aux deux.

8 Andreï Fadin ${ }^{8}$ définit ces conflits comme n'étant ni des guerres (faiblesse des protagonistes, alternance de combats et d'accalmies) ni des révolutions (maintien des hiérarchies sociales et des élites). Il y voit plutôt une réorganisation de l'espace exsoviétique par l'établissement d'une nouvelle hiérarchie nationale et étatique, unique par son ampleur au vingtième siècle. Il insiste surtout sur la diffusion, au-delà du conflit armé lui-même, de la violence qui devient dans les zones concernées partie intégrante de la vie quotidienne et de la culture politique. Une génération de " déracinés " s'est formée, socialisée par la violence, sans autres repères, incapable de retourner à la vie civile et d'abandonner le statut acquis par exemple dans les différentes milices. Ces conflits sont largement le fruit d'une lutte pour l'appropriation des ressources entre les anciennes élites locales et une contre-élite surgie à la fin des années quatre-vingt. Reposant sur l'utilisation comme ressource d'un sentiment national et d'une revendication d'identité réels, ces conflits ont basculé dans la violence, voire dans la guerre civile comme en Géorgie.

L'approche qui prévaut généralement dans l'analyse des conflits interethniques est une approche en termes de " points chauds "9, qui privilégie le monitoring des conflits ouverts ou potentiels, qu'il s'agisse des Nouveaux Etats Indépendants ou de la Fédération de Russie elle-même. Un atlas récent de la Russie ${ }^{10}$, par ailleurs remarquable de précision et de qualité, présente cent soixante-dix conflits en Fédération de Russie. En se penchant sur le détail des conflits recensés et cartographiés, on constate que la plupart sont des revendications politiques portées essentiellement par des responsables politiques locaux ou régionaux, plus rarement des mobilisations sociales. La violence est " surveillée ", décrite ${ }^{11}$, rarement pensée.

10 La guerre en Tchétchénie ${ }^{12}$

11 Dans le conflit tchétchène, première guerre à se dérouler sur le territoire russe, la Russie post-communiste a joué plusieurs cartes de son avenir. Après plusieurs années de conflits avec la République rebelle qui avait déclaré son indépendance dès 1991, après avoir tenté de jouer des oppositions internes à la République pour renverser le Général Doudaev, dont il s'était pourtant accommodé pendant plusieurs années, le pouvoir russe a choisi d'utiliser la force en décembre 1994.

12 Sur les causes du conflit, de nombreuses hypothèses circulent sans qu'il soit possible de mesurer la pertinence de chacune : guerre du pétrole dont l'enjeu serait, entre autres, le contrôle des oléoducs traversant la Tchétchénie depuis la mer Caspienne; guerre religieuse, argument que l'on a vu poindre depuis peu en Russie, où l'on présente 
parfois les Tchétchènes comme des " fondamentalistes "; guerre anticoloniale, qui prolonge un siècle et demi de résistance et pourrait s'étendre à d'autres peuples voisins; guerre des chefs à Moscou, qui se manipuleraient les uns les autres pour le règlement de conflits d'intérêts ou pour une surenchère nationaliste à destination de l'opinion. Guerre d'aveuglement, pourrait-on ajouter, de la part d'un pouvoir tétanisé par la possibilité d'une réproduction du scénario de la fin de l'URSS à l'échelle de la Fédération de Russie.

13 Le conflit en Tchétchénie illustre une logique d'engrenage où, du côté du pouvoir à Moscou, le sens, à la fois comme direction et comme signification, se perd. Ordres et contre-ordres, motivations peu définies et différentes pour chacun des protagonistes, enjeu à la fois direct et indirect, le théâtre des opérations en Tchétchénie n'est qu'une manière de régler d'autres conflits, de procéder à des redistributions de pouvoirs et de ressources à l'intérieur de l'appareil politique et économique du pays. La précision de Jacques Sapir quand il écrit " qu'il n'y a pas de guerre de Tchétchénie mais une guerre en Tchétchénie " ${ }^{13}$ renvoie à l'ensemble de ces facteurs mais peut laisser penser à une pure instrumentalisation. Or, le conflit tchétchène offre à la fois la combinaison de toutes les formes de violence que l'on a pu décrire, mais est aussi un conflit spécifique, qui témoigne à la fois du poids de l'histoire et de la montée de nouvelles revendications identitaires et nationales dans l'espace de l'ex-URSS.

Il est trop tôt pour faire le bilan d'une guerre qui pendant plus de dix-huit mois, a particulièrement touché la population civile et les jeunes appelés russes du contingent, détruit presque entièrement la capitale tchétchène. Le siège des villes et des villages s'est accompagné de violations massives des droits de l'homme ${ }^{14}$ et la criminalisation de l'armée russe ${ }^{15}$ a été maintes fois soulignée. Sur le territoire russe, le conflit a donné lieu à plusieurs épisodes terroristes.

Les accords qui ont été signés, après négociations entre les indépendantistes tchétchènes et le Général Lebed, limogé depuis, sont à peu près respectés jusqu'à ce jour. Ils ne garantissent pas pour autant une paix durable; les désaccords sont nombreux sur sa valeur juridique et surtout, leur avenir dépendra de qui a intérêt à faire la guerre - ou la paix - dans cette région. La conduite du processus par le Général Lebed, seul à être considéré comme interlocuteur légitime par les Tchétchènes, a joué un rôle capital. Au-delà du paradoxe que constituent des accords de paix obtenus par un militaire là où les civils ont échoué, cet élément montre qu'une sortie de la violence peut se faire lorsqu'une rupture se produit : ici l'arrivée d'un homme, non seulement respecté pour sa personnalité et son passé, mais largement étranger aux causes et aux mécanismes du conflit, ce qui lui permet d'être reconnu comme interlocuteur.

16 La rapidité avec laquelle on a " fait la paix " peut aussi être interprétée comme un signe supplémentaire du caractère purement instrumental et " moscovite " de cette guerre, de même que la décision du gouvernement de retirer ses dernières troupes, annoncée à grand renfort de publicité qui vient à point nommé pour deux raisons : montrer à l'opinion que le Général Lebed n'est pas le seul à vouloir la paix et à être capable de l'obtenir ; faire oublier opportunément les accusations de corruption qui ont ébranlé les nouveaux hommes forts du Kremlin et notamment le chef de l'administration présidentielle Anatoly Tchoubaiis.

17 Ce type de raisonnement est séduisant parce qu'il semble d'une part coller à la réalité des faits et en fournir une clé universelle, et d'autre part conforter une analyse des décisions du pouvoir en Russie dans les termes du complot et de la seule lutte de clans. 
Il nous semble insuffisant pour plusieurs raisons. Il accorde trop de place au système, vu de Moscou. Il sous estime la capacité d'action des responsables et des combattants tchétchènes alors que leur part est loin d'être négligeable dans la décision de négocier et de parvenir à un accord. D'autre part, ce type de raisonnement méconnaît l'incertitude qui règne dans les plus hauts niveaux du pouvoir et le degré de hasard qui peut influer sur les décisions les plus importantes. Sociologitcheskie issledovaniâ ${ }^{18}$, attirait l'attention sur l'apparition d'un phénomène de 
" bandes "19: des groupes de cinquante à cent adolescents descendant sur Moscou en train depuis la province, pour y commettre des actes de délinquance plus facilement dissimulables. On y décrivait leurs modes d'organisation: " comptoirs ", rassemblant ponctuellement des jeunes étudiant ou habitant au même endroit ; bandes criminelles, plus organisées, qui mêlent adolescents et adultes avec une " caisse " pour aider financièrement les prisonniers, les malades et la famille de ceux qui sont morts et dont certaines portent le nom de "groupes du risque "; Chelukha pour les plus jeunes adolescents de quatorze, quinze ans. L'auteur tentait de formuler leur " idéologie " : " [La bande] est pour les véritables hommes, pour qu'ils se sentent des gens dans leur pleine valeur. Comment vivent donc les adolescents? A la maison, les parents les grondent, à l'école ce sont les professeurs. Le " comptoir les protège " et leur donne la possibilité de se tester eux-mêmes, en jouant aux jeux des adultes avec des règles très dures "20. Aujourd'hui, la violence n'est pas constituée en objet social et s'est comme diluée entre le marché et l'anomie ${ }^{21}$. Ses manifestations sont renvoyées à l'histoire et aux changements systémiques, plus souvent encore à la sphère de la psychologie et des comportements individuels. Elle est cependant constamment redoutée et annoncée. Ainsi un sociologue du ministère de l'Intérieur en Sibérie n'imagine pas que la violence urbaine " ne puisse pas advenir dans un proche avenir si les 'nouveaux Russes' continuent à se faire construire des luxueux cottages dans les terrains libres entre deux cités-dortoirs "22. En même temps, comme par crainte de ses propres paroles, il explique l'absence de ce type de violence par une grande faculté d'adaptation sociale en Russie : " Les gens se sont adaptés : les pauvres à la pauvreté, les riches à la richesse... ". On peut mentionner, moins pour sa plausibilité que pour ce qu'elle révèle des perceptions de l'état de la société parmi les autorités, une hypothèse avancée ${ }^{23}$ lors des attentats dans les transports de Moscou de juin et juillet 1996, selon laquelle un déséquilibré pourrait en être l'auteur, notamment parce que deux des attentats avaient été commis le 11 du mois : " Des cas de ce type sont courants dans une société qui se trouve dans un si mauvais état socio-psychologique (...) Supposons que quelqu'un doive être payé le 11 de chaque mois mais qu'il n'ait pas été payé depuis longtemps. Il va décider de 'célébrer' son jour de paye de cette terrible manière (...) ". Ce raisonnement, qui renvoie à une société " malade ", revient à faire l'hypothèse d'une violence aux causes " sociales ", mais où seuls des individus se manifestent. L'hypothèse est fantaisiste mais l'un des problèmes sociaux majeurs de la Russie actuelle est mis en évidence : les retards accumulés dans le paiement des salaires conduisent effectivement à des situations tragiques pour beaucoup d'individus. Ce raisonnement reflète plus profondément l'étonnement et l'incompréhension face à l'absence de réactions sociales sur ce problème, qu'il s'agisse de mobilisations classiques (grèves, manifestations) ou d'explosions de violence. La mise en relation d'un problème qui devrait provoquer de la violence avec des faits réels mais inexpliqués est un pas d'autant plus facile à franchir que les médias ne cessent de prédire ces explosions.

Autre exemple, en mars 1996, inquiet des proportions prises par les fraudes dans les bus, malgré la mise en place de receveurs (essentiellement des femmes), le service des transports urbains de la ville d'Omsk décide de mener une grande opération de contrôle. Craignant des troubles et des agressions contre les contrôleurs, il décide de faire appel à la police pour protéger ces derniers pendant les contrôles. Aucun incident n'a été signalé de toute la journée... On est là-aussi dans le cas d'une violence fantasmée, mais possible. Si elle se manifestait, elle pourrait être considérée comme logique, voire contenir des motivations sinon légitimes, du moins compréhensibles. 
C'est donc son absence qui est incompréhensible, y compris pour les autorités chargées de la prévenir ou de la punir, comme si la Russie défiait des " lois sociologiques " éprouvées ailleurs.

Le discours est fréquent parmi les intellectuels en Russie sur la menace potentielle du lumpen : ces couches déclassées et nostalgiques du régime soviétique seraient prêtes à la révolte, pour peu qu'un leader surgisse ou que leurs conditions de vie descendent en deçà d'un certain seuil. Ce discours qui fait une large part au mépris n'est pas sans rappeler celui des " classes dangereuses " dans l'Europe de la Révolution industrielle. Aucun leader n'a jusqu'à présent entraîné de masses vers une révolte sociale et les partis de la mouvance communiste en sont très loin.

En revanche, on pourrait considérer comme porteuses de significations sociales les violences physiques et psychologiques ayant cours dans les prisons et les casernes, des pratiques qui relèvent des caractéristiques " naturelles " $d$ 'institutions répressives et de comportements pathologiques, mais aussi d'une l'indifférence de la société à l'égard d'une catégorie, les prisonniers ou les appelés, qui refléterait l'indifférence et le peu d'exigences que la société parvient à formuler pour elle-même. Ou bien avancer l'hypothèse que les suicides, qui ont notamment frappé l'opinion à l'automne 1996, pourraient être une manifestation de la violence sociale, même s'il s'agit d'une violence contre soi ${ }^{24}$.

La criminalisation qui investit à la fois la société et l'Etat rend problématique les distinctions pertinentes en d'autres lieux entre la violence de l'Etat et la violence sociale ou politique, ou encore entre la violence instrumentale, froide, et la violence expressive, chaude. Et si l'on devait reprendre ce type de distinction, on serait tenté, au risque de pousser le trait, d'inverser les rôles : la violence à laquelle ont recours les groupes criminels qui commanditent des meurtres ou font exploser une bombe dans un cimetière lors $\mathrm{du}$ rassemblement d'anciens d'Afghanistan, est beaucoup plus " instrumentale ", calculée, que l'arbitraire et le sadisme qui règnent dans les prisons ou dans les casernes des appelés. L'utilisation calculée d'une violence qui restera impunie dans les milieux d'affaires ressort d'un calcul coût/avantage certainement plus " intéressant " que la violence manifestée pendant la guerre en Tchétchénie, dans un conflit où l'Etat se discrédite sans en venir à bout.

Les représentations sociales et politiques de la violence

Il s'agit ici de s'intéresser aux perceptions de la violence qui alimentent le sentiment d'insécurité grandissant de la population et de tenter de l'articuler avec le niveau de violence réelle dans la société; il s'agit aussi de revenir sur certaines attitudes culturelles et politiques et de montrer que la relation qu'entretient la société avec la violence est ambiguë, et faite de rejet tout autant que de tolérance.

" La Russie que nous avons perdue "25

Pendant la période soviétique, l'idéologie de la lutte des classes et l'influence pernicieuse d'un Occident en proie à la décadence tenait lieu pour le pouvoir de discours sur la violence. Lors d'une réunion du comité régional du Parti de la région de Sverdlovsk (dans l'Oural) en 1982, les responsables invoquent l'influence négative de films ou feuilletons occidentaux (citant par exemple Fantômas) sur des adolescents fragiles amenés à commettre des actes de hooliganisme ${ }^{26}$.

Mais dans l'ensemble, dans un système qui, même affaibli, reposait en partie sur la force de la propagande, le sentiment de sécurité, d'une vie sociale tranquille et d'une 
éradication progressive de la criminalité était largement répandu. Aujourd'hui, " les mythes se sont entrecroisés avec la réalité. Une attitude confortable et illusoire vis-àvis des statistiques de la criminalité a laissé place à une panique 'informée' "27. Aujourd'hui, on peut parler, en regard de la démonopolisation de la violence, d'une démonopolisation de la peur. A la crainte omniprésente mais unidirectionnelle de la répression politique et policière émanant des services de sécurité soviétique, a succédé une différenciation des sources et des cibles de la violence ${ }^{28}$.

Le sentiment d'insécurité, massif en Russie aujourd'hui, est mesuré par de nombreuses enquêtes et sondages. Au niveau national, une enquête du VNII ${ }^{29}$ sur l'ensemble de la Russie en 1995 fait état d'un sentiment d'angoisse face à la criminalité pour $42 \%$ de la population, et d'inquiétude pour $47 \%$. A Omsk ${ }^{30}$, les deux préoccupations principales sont le paiement irrégulier des salaires $(59 \%)$ et la hausse de la criminalité $(53 \%)$. Malgré la peur d'être personnellement victime d'une agression, la criminalité passe après les problèmes sociaux lorsque la question porte sur le monde proche. Des sondages récents du VTSIOM montrent des écarts dans les perceptions : les inquiétudes principales " dans sa région " sont le non-paiement des salaires et le chômage.

Même si le monde de la criminologie en Russie reste traditionnel dans on approche des phénomènes, avec des représentations morales et psychologisantes (qui ont remplacé l'idéologie), on observe des tentatives de la part de certains criminologues pour renouveler l'approche, prenant acte de la crise de leur discipline face à la montée de la criminalité et de ses nouvelles manifestations. Il en ressort une conception " naturaliste " de la violence mélangée à une analyse sociale générale post-moderne, et à des références à la théorie des conventions (le crime est une convention) ainsi qu'aux analyses d'Howard Becker sur la déviance ${ }^{31}$. Mais la démarche a le mérite de dépasser les approches traditionnelles du phénomène (passées du scientisme soviétique à des conceptions essentialistes en termes de perte de spiritualité, ou de caractère russe), en se donnant comme objectif de trouver une nouvelle théorie et en mettant l'accent sur l'inefficacité des formes actuelles du " contrôle social ", à savoir le système judiciaire et carcéral.

On peut mentionner la catégorie " hooliganisme " (Khuliganstvo), délinquance de rue à mi-chemin entre incivilités et agressions, commise en règle générale par des jeunes et des adolescents, très souvent en état d'ivresse. Selon les statistiques, ou selon les auteurs, les actes de " hooliganisme " peuvent être ou non classés parmi les actes de violence contre les personnes. Mais ils sont très fréquemment mentionnés comme la catégorie la plus en progression et l'une des plus menaçante car susceptible de toucher n'importe quel citoyen, et de basculer dans la violence : " La grossièreté, l'ivrognerie, les batailles de rues sont devenues des manifestations si courantes que les policiers n'y prêtent même plus attention (...) Les petits spéculateurs sont partout dans les villes et se considèrent comme les maîtres absolus de la rue (...) Le grand risque qu'il y a à faire preuve d'indulgence à leur égard est de créer dans la société une atmosphère de grossièreté, d'atteinte permanente à la dignité humaine. Un petit délinquant (khuligan) se transforme facilement en tueur, l'impunité renforçant ses orientations antisociales "132.

Le hooliganisme apparait ainsi en forte correspondance avec les craintes de la société, symbolisant l'écroulement de l'ordre (la propreté des rues et des bâtiments aujourd'hui dégradés, voire " taggés "), de la morale (en opposition aux comportements " déviants " des jeunes), de la sécurité et de la stabilité générale assurées auparavant par le système 
et dont ces " comportements antisociaux " viennent faire encore plus ressentir, en creux, l'absence et la nostalgie.

S'agit-il de la découverte d'un phénomène objectif ou de celle d'un sentiment ? Certains n'hésitent pas à affirmer qu'il " était tout aussi dangereux de se promener le soir dans les rues il y a dix ans mais que simplement, on n'en parlait pas "33, et à invoquer la mémoire courte de la société qui aurait comme oublié l'époque de la répression massive.

D'une discussion collective avec des journalistes traitant des problèmes de criminalité dans la ville d'Omsk, sort un tableau des plus confus, toute la question tournant sur la comparaison possible entre la période soviétique et la période actuelle pour le niveau de violence et de criminalité. Un consensus se dégage pour affirmer que le niveau était beaucoup plus élevé que ne le montraient les statistiques, et tous peuvent citer des batailles entre bandes dans des quartiers de semi bidonvilles après la guerre, des règlements de compte au couteau dans les cages d'escalier ou des jeunes drogués braquant les pharmacies dans les années soixante-dix. Tous s'accordent aussi à souligner la corruption de la police qui, en cas de violence entre jeunes, " propose parfois de l'argent aux parents pour qu'ils retirent leur plainte ", et évoquent le facteur aggravant de l'institution pénitentiaire.

40 La crise de confiance vis-à-vis des institutions en charge de la sécurité

41 La crise de confiance vis-à-vis des organes de maintien de l'ordre policière est manifeste. Non seulement la police ne respecte pas les droits de la personne ${ }^{34}$, mais elle ne répond pas à ce qu'on attend d'elle et les citoyens s'en détachent, la tendance pouvant prendre des proportions très importantes dans certaines villes : la moitié des personnes interrogées n'iront pas à la police si elles sont victimes d'une agression ou d'un crime, $49 \%$ pensent qu'en cas de problème, la police ne les aidera pas, $35 \%$ évaluent négativement le travail de la police, $20 \%$ considèrent qu'elle est liée au crime organisé. A propos de la violence domestique, I. Antonian ${ }^{35}$ remarque que la défiance à l'égard des forces de maintien de l'ordre n'est pas sans fondement, car celles-ci ont très peu de chance de tenir compte des plaintes.

42 Les défauts principaux qui sont reprochés sont l'incompétence et l'indifférence aux citoyens. Un échantillon de policiers qui figurait dans l'enquête donnait à peu près les mêmes réponses. Aspect plus surprenant, mais qui témoigne encore une fois de la privatisation de la gestion de la sécurité : nombreux sont ceux qui seraient prêts à payer pour que la police soit plus efficace, ou à l'aider eux-mêmes. Un rapport du VTSIOM établi en 1990 à partir de plusieurs sondages ${ }^{36}$ montrait déjà une sérieuse préoccupation en matière d'insécurité et une défiance presque aussi grande vis-à-vis de la police.

43 La même méfiance est observée vis-à-vis de la justice, notamment en raison de l'impunité des criminels, souvent dénoncée par les médias, mais aussi par les spécialistes de criminologie qui remarquent une tendance, inverse à la progression de la criminalité, à la baisse continue et sensible du nombre de condamnations ${ }^{37}$. A propos de la peine de mort ${ }^{38}$, les spécialistes mettent en avant une double disproportion : dans le rapport entre les condamnations à mort et le nombre de meurtres en Russie par comparaison avec les Etats-Unis d'une part; entre le nombre de condamnations à mort et un taux de grâces " trop élevé ", par rapport à ce que peut supporter la société d'autre part ${ }^{39}$. Sur cette question, il semble exister un quasi-consensus pour considérer que la société n'est pas prête, y compris parmi des personnalités libérales, à l'exception 
de quelques organisations des Droits de l'Homme clairement abolitionnistes. Les causes de cette situation seraient à rechercher à la fois dans l'héritage soviétique, qui a pendant des décennies chargé les mentalités de violence, qu'il s'agisse de la lutte des classes, de la guerre, ou de l'idéologie totalitaire, mais aussi dans la vague objective de criminalité que connaît le pays et dans la faiblesse de l'Etat à y répondre : " Elevée dans la violence, la cruauté et le crime, la société exige la même cruauté envers les criminels "40. Une attitude qui montre la difficulté des représentations, prises entre le passé et le présent.

Les promesses de campagne contre la criminalité organisée sont discréditées, en apparaissant comme de simples effets d'annonce. De plus, ce discrédit est générateur de violence de la part de groupes qui cherchent à dissuader les responsables politiques de mettre en oeuvre des projets qui, pour la majorité de l'opinion, n'ont de toutes façon pas la moindre chance d'aboutir. Mais on peut aussi faire l'hypothèse que les groupes criminels et leurs réseaux de couverture et de protection au sein du pouvoir central et des pouvoirs locaux n'ont pas intérêt à utiliser une violence terroriste génératrice d'instabilité ou de réactions politiques imprévues du pouvoir, leur développement nécessitant au contraire la stabilité. Il est probable par ailleurs que l'opinion perçoit, même de manière diffuse, que la violence froide et calculée à laquelle ont recours les groupes criminels n'est pas, pour le " simple citoyen ", plus dangereuse que d'autres formes. On n'ira pas jusqu'à dire que ces réseaux bénéficient d'un capital de sympathie dans l'opinion; à la différence de ce que l'on observe dans d'autres expériences, ils n'adoptent pas un comportement de bienfaiteurs. Mais les règlements de compte semblent provoquer des réactions ambivalentes combinant dans l'opinion : une montée du sentiment d'insécurité, doublée d'émotion si la victime était une personne publique reconnue et estimée ; une certaine indifférence à ce qui apparaît comme une violence endogène ne concernant qu'un groupe bien déterminé de la population; enfin, ce que l'on pourrait qualifier d'effet " Robin des Bois ", tendant à justifier ce type d'assassinat, au motif " qu'il n'y a pas de fumée sans feu " - les commanditaires commettent une bonne action en éliminant un criminel que la police inefficace et corrompue n'aurait pu arrêter. Des criminologues ${ }^{41}$ ont observé le mélange de ces réactions à la suite de l'assassinat à Omsk d'Oleg Tchertov, jeune et brillant directeur d'une entreprise de pneus, député régional depuis novembre 1994 et bien perçu par l'opinion. Passé le choc des premiers moments, le doute et la suspicion se sont installés pour aboutir à l'opinion générale qu'il s'agissait d'un règlement de compte et que s'il y avait compte à régler, c'est que la victime devait être elle-même, à un degré ou à un autre, liée à des activités criminelles.

L'opinion publique a peur des violences sociales et politiques et semble vivre dans une tension d'autant plus forte qu'elles ne se produisent pas: les sondages font régulièrement état d'attitudes considérant comme probables les grèves, les meetings contre le gouvernement ou les tentatives de coup d'Etat. Ces dernières semblent être l'objet d'une attention particulière dans les milieux intellectuels, notamment à Moscou. En revanche, la perception d'une menace directe est ambivalente. Selon un monitoring régulier du ministère de l'Intérieur à $0 \mathrm{msk}^{42}$, on redoute d'être au chômage $(38 \%$ en 1995) plus que d'être agressé ou cambriolé ( $28 \%$ et $29 \%$ ) ou d'être victimes de " désordres de masse ou de pogroms " (14\%). En revanche, et la comparaison vaut d'être soulignée, en 1993, $64 \%$ ressentaient un fort sentiment d'angoisse et d'insécurité pour leur famille, ce chiffre étant tombé à $40 \%$ en 1995, avec $2 \%$ et $6 \%$ de réponses négatives. Malgré un relatif apaisement, ces chiffres restent très forts. Reliés à la faible 
perception de menaces concrètes, ils nous semblent conduire à une hypothèse : c'est bien un sentiment d'angoisse et d'insécurité qui domine les représentations sociales, mais ce sentiment n'a qu'en partie à voir avec la criminalité et l'insécurité objective. En revanche, les médias, l'institution policière, le pouvoir politique participent largement à la construction d'un lien direct entre sentiment d'insécurité et criminalité.

\section{NOTES}

1. J. Michael Waller, Victor J. Yasmann, " Russia's great criminal revolution : the role of the security services " in Journal of Contemporary Criminal Justice, op. cit., p. 276-297. 2. Cf. bulletin régulier, Prava celoveka i sluzby bezopasnosti (Les Droits de l'Homme et les services de sécurité), Fondation Glasnost, paraît depuis janvier 1996.

3. " Building soviet militaries ", suite d'articles parus dans Transition (Prague), vol. 2, n' 16, 9 août 1996, p. 4-17, 64.

4. Graham H. Turbiville Jr, " Organized crime and the Russian armed forces ", dans Transational Organized Crime, vol. 1, nº 4, Hiver 1995, p. 57-104.

5. Ainsi sont définis officiellement en Russie les pays limitrophes de la Russie, anciennes Républiques de l'Union soviétique.

6. Sur ce point particulier, mais aussi sur l'ensemble des questions relatives aux forces armées, cf. Jacques Sapir, Le Chaos russe, op. cit., notamment les chapitres 6 et 7.

7. Selon l'expression de Charles H. Fairbanks Jr, " The postcommunist wars " in Journal of Democracy, vol. 6, n 4, oct. 1995, p. 18-34. Cet article, qui dépasse le cadre de la Russie, recense et analyse touts les formes de privatisation militaires et para-militaires dans les conflits de l'espace ex-communiste.

8. Andrej Fadin, " Vlast' cerez krov' : put' naverh (Socialnyj smysl' postsovetskyh vojn " [Le pouvoir par le sang : une sortie vers le haut? (La signification sociale des guerres post-soviétiques] Znamâ, 1993, n² 2, p. 176-185.

9. Georgij Vatchnadze, Gorâcie tocki Rossii [Les points chauds de Russie], Moscou, Kniga, 1993, qui, par points chauds, entend aussi bien les revendications régionalistes de régions riches en ressources naturelles que la "deuxième guerre du Caucase" annoncée ; ou le monitoring effectué chaque fois par des équipes de l'Institut d'ethnologie de l'Académie des sciences sur les conflits de l'ex-URSS, en lien avec des organisations des Droits de l'Homme qui recensent les violations des droits et tentent un travail de prévention et de médiation dans ces conflits.

10. Atlas de la Russie et des pays proches, Paris, Institut Reclus, La Documentation française, 1996.

11. Pour une description détaillée de tous les conflits post-soviétiques et des violences et crimes qu'ils ont occasionnés, voir aussi, Viktor V. Luneev, " Prestupnost' v meznacional'nyh konfliktah " [La criminalité dans les conflits interthniques] in Sociologiceskie issledovaniâ, 1995, n 4, p. 103-11, n 5, p. 99-109.

12. Le conflit en Tchétchénie aurait pu à lui seul faire l'objet d'une recherche. On a choisi ici de ne pas le traiter en détail. Pour des aspects plus généraux ou historiques, on peut voir Patrick Karam et Thibaut. Mourgues, Les guerres du Caucase : des Tsars à 
la Tchétchénie, Paris, Perrin, 1995 ; Marie Bennigsen Broxup, " Le Caucase du nord : conflit etchnique ou crise coloniale " in Le Caucase postsoviétique : la transition dans le conflit, Bruxelles, Bruylant, Paris LGDJ, 1995 (ainsi que les autres publications de Marie Bennigsen) ; Alexandre Popovic et Gilles Veinstein, Les voies d'Allah : les ordres mystiques des origines à aujourd'hui, Paris, Fayard 1996, le chapitre de Chantal Lemercier-Quelquejay sur les traditions soufies dans le Caucase et particulièrement les deux ordres concurrents en Tchétchénie ; Robert F. Baumann, " Historical perspective on the conflict in Chechnia " in Low Intensity Conflict and Law Enforcement, vol. $4 \mathrm{n}^{\circ} 1$, été 1995, p. 119-132 ; L. S. Perepelkin, " Cecenskaâ respublika : sovremrennaâ social'nopoliticeskaâ situaciâ " in Etnograficeskie obozrenie, janv-fév. 1994, p. 3-15. Sur le conflit, outre la presse russe et internationale, Nina Bachkatov et Andrew Wilson, " Tchétchénie, : histoire d'un conflit ", dans Les dossiers du Grip, nº 1, 1995 ; Emil Pajn et Aleksandr Popov, " Cecenskaâ politika v Rossii s 1991 do 1994 " [La politique tchétchène de la Russie de 1991 à 1994] in Mirovaâ èkonomika i mezdunarodye otnošeniâ, $n^{\circ}$ 5, 1995, p. 19-32. ; " The future of Russia " in Comparative Strategy, vol. 15, n 1, 1996, plusieurs articles ; M. Fejgin, " Vtoraâ kavkazskaâ vojna " (La deuxième guerre du Caucase), Novyj mir, n 12, 1995, p. 159-171. ; Jacques Sapir, Le chaos russe, op. cit. ; Nicholas M. L. Bovay, " The Russian armed intervention in Chechnya and its human rights implications " in International Commission of Jurists : the Review ; n 54, 1995, p. 29-57 ; "Tchétchénie : une guerre coloniale ", suite d'articles dans Politique internationale, $\mathrm{n}^{\circ}$ 67, printemps 1995, p. 47-153 ; Géopolitique du Caucase, Hérodote, $\mathrm{n}^{\circ}$ 81, avril-juin 1996. Sur l'opinon publique, M. Haney, "Russia's first télévision war : public opinion in the crisis ", Transition, 14. 4.1995 ; L. Gudkov, " God Cecenskoj vojny v obšcestvennom mnenii Rosii " [Un an de guerre en Tchétchénie dans l'opinion publique] in Druzba narodov (L'amitié des peuples), n² 2, 1996, p. 160-168, ainsi qu'en Russie, de nombreuses publications émanant d'organisations des droits de l'homme : le Comité des mères de soldats, la Fondation Glasnost, Memorial, une chronique régulière publiée les premiers mois de la guerre par plusieurs d'entre elles, "Ne vidit', ne slyšat', ne ponât'" (Ne pas voir, ne pas entendre, ne pas comprendre), etc.

13. Jacques Sapir, Le chaos russe, op. cit.

14. De nombreux témoignages et documents ont été publiés après plusieurs de ces épisodes, notamment les sièges de Smashki et Gudermes, ainsi que les bombardements de Pervomajskoe. cf. notamment Fondation Glasnost " Vojna v Cecnii : mezdunarodnyj tribunal ", Moscou, 1996 ; Memorial, Vsemi imeûšcimmisâ sredstvami, (Operation du MVD dans le village de Samashki), Moscou, 1995 et U nas ne okazalos' podvodnoj lodki : Hronika zadymleniâ, Kizlâr-Permomajskoe 9-26 ânvarâ 1996, Moscou, 1996.

15. Au fur et à mesure que le conflit se prolongeait, les autorités russes ont utilisé de plus en plus d'engagés volontaires sous contrat, parmi lesquels, des détenus de droit commun bénéficiant ainsi de remises de peine, en fonction de la durée de leur engagement. De nombreux cas de racket des habitants des villages assiégés ont été rapportés.

16. Ce sont les groupes criminels qui utilisent ces méthodes (favorisées par les nombreux explosifs en circulation) contre les bâtiments et de plus en plus contre les personnes. On comptait en 1995, cent trente attentats à l'explosif à Moscou et dans sa région. Les tueurs à gage utilisent régulièrement les engins explosifs, comme lors de l'assassinat du journaliste Dmitry Kholodov.

17. Propos du maire de Moscou, Youri Loujkov, cités dans Le Figaro du 13 juillet 1996 et Le Monde du 15 juillet 1996. 
18. Recherches sociologiques, revue dont les articles étaient souvent à cette période des "poissons-pilotes" sur des questions sociales délicates ou auparavant interdites d'expression publique. L'article mentionné est de I. Y. Petelin, " Organizovannaâ prestupnost' nesoveršennoletnyh " [Le crime organisé chez les mineurs] in Sociologiceskie issledovaniâ, n 9, 1990, p. 93-98.

19. Voir aussi, un entretien avec A. Arendar, Etudes polémologiques, $n^{\circ} 50$, février 1989 , qui analyse le phénomène dans la ville de Tchéliabinsk (sud de l'Oural).

20. Y. Petelin, op. cit., p. 93.

21. On peut ici appliquer aux représentations et aux significations de la violence des conclusions tirées par ailleurs sur l'ensemble de la société. Cf. notamment Alexis Berelowitch, Michel Wieviorka, Les Russes d'en-bas, Paris, Seuil, 1996.

22. Entretien avec O.V., responsable du département de sociologie de la filiale pour la Sibérie occidentale du VNII (Zapadno-sibirskij otdel' Vserossijskogo Naucnogo Issledovatel'skogo Instituta), Omsk, 22 avril 1996. Le VNII est le département d'enquêtes, sondages et statistiques qui dépend directement du ministère de l'Intérieur, à Moscou et dans les directions régionales.

23. Propos du Colonel G. Vanine, recueillis par Les Nouvelles de Moscou, 18 juillet 1996. 24. Ils ont été directement mis en relation avec le désespoir né de la situation sociale, comme en témoignent en tout cas certains écrits ; Ils ont touché des personnes appartenant à tous les milieux, le célèbre physicien nucléaire Netchaev à Tcheliabinsk, l'ouvrier non payé depuis des mois d'une usine de Tagan-Rog ou le directeur d'un sovkhoze. cf. " Khronika neob'âvlennogo samoubijstvo " [Chronique d'un suicide non annoncé] in Izvestiâ, 2 novembre 1996, p. 2.

25. Titre d'un film de Stanislav Govorukhine qui traite en particulier du développement de la criminalité, avec un discours à la fois sécuritaire et nostalgique.

26. Archives du Comité régional du Parti de la région de Sverdlovsk. Fonds en cours d'exploitation par Gilles Favarel-Garrigues.

27. " Ugolov'naâ Rossiâ ", Izvestiâ, in Russian Politics and Law, juillet-août 1995, p. 8-42.

28. Voir notamment sur ce point les analyses les plus récentes de Vladimir Schlapentokh.

29. VNII, voir note 73.

30. Monitoring régulier du VNII, Omsk, 1995.

31. Âkov Gilinskij, " Some problems of contemporary crime and criminology ", Conférence internationale "Comportement déviant et contrôle social", op. cit., p. 63 sq. 32. Ûri M. Antonân, Zestokost' v našej zizni [La cruauté dans notre vie], op. cit. , p. 57. 33. V. K. Kantor, " Nasilie kak provokacia civilizacionnyh sryvov v Rossii " [La violence comme cause des coups d'arrêts à la civilisation en Russie] in Rossijsko-germanskij dialog : nasilie v posttotalitarnyh obšcestvah [Dialogue germano-russe : la violence dans les sociétés post-totalitaires] Voprosy filosofii, $n^{\circ} 5,1995$, p. 3-47

34. Sondage VNII, 1995 : 3 \% des personnes interrogées sur l'ensemble de la Russie citent la police comme institution représentant les droits. $30 \%$ répondent "personne" ; $5 \%$ pensent que la police défend les intérêts du citoyen, 20 \% "ses propres intérêts", 19 \% "les intérêts de groupes particuliers".

35. Zestokost' v našej zizni [La cruauté dans notre vie] op. cit., p. 60.

36. Rapport : Enquête sur l'attitude l'opinion publique en RSFSR par rapport à la criminalité et au travail des forces de maintien de l'ordre, Moscou, VTSIOM, 1990.

37. Izmeneniâ prestupnosti v Rossii, op. cit., p. 10. 
38. Avec une recrudescence des exécutions en 1995, après plusieurs années de relative clémence, la Russie est aujourd'hui accusée par les organisations des Droits de l'Homme de ne pas respecter le moratoire auquel elle s'était engagée lors de l'acception de sa candidature par le Conseil de l'Europe. Quatre-vingt-six exécutions auraient eu lieu en 1995, contre quatre en 1993 et en 1994.

39. Vitaly Kvashis : " Capital punishment in Russia : current tendancies ", communication présentée à l'Academy of Criminal Justice Sciences, annual meeting, Las Vegas, 12-16 mars 1996.

40. Idem.

41. Entretien avec V. S, E. K, et N. B., professeurs à l'Ecole supérieure de la police, Omsk, avril 1996.

42. Monitoring régulier du VNII, Omsk, 1992, 1993, 1995.

INDEX

Index géographique : Russie

Mots-clés : mafias et crime organisé, violence 